\title{
Concurrent System Programming with Effect Handlers
}

\author{
Stephen Dolan ${ }^{1}$, Spiros Eliopoulos ${ }^{3}$, Daniel Hillerström ${ }^{2}$, Anil Madhavapeddy ${ }^{1}$, \\ KC Sivaramakrishnan ${ }^{1}(\bowtie)$, and Leo White ${ }^{3}$ \\ ${ }^{1}$ University of Cambridge \\ 2 The University of Edinburgh \\ 3 Jane Street Group
}

\begin{abstract}
Algebraic effects and their handlers have been steadily gaining attention as a programming language feature for composably expressing user-defined computational effects. While several prototype implementations of languages incorporating algebraic effects exist, Multicore OCaml incorporates effect handlers as the primary means of expressing concurrency in the language. In this paper, we make the observation that effect handlers can elegantly express particularly difficult programs that combine system programming and concurrency without compromising performance. Our experimental results on a highly concurrent and scalable web server demonstrate that effect handlers perform on par with highly optimised monadic concurrency libraries, while retaining the simplicity of direct-style code.
\end{abstract}

\section{Introduction}

Algebraic effect handlers are a modular foundation for effectful programming, which separate the operations available to effectful programs from their concrete implementations as handlers. Effect handlers provide a modular alternative to monads $[25,35]$ for structuring effectful computations. They achieve the separation between operations and their handlers through the use of delimited continuations, allowing them to pause, resume and switch between different computations. They provide a structured interface for programming with delimited continuations [10], and can implement common abstractions such as state, generators, async/await, promises, non-determinism, exception handlers and backtracking search. Though originally studied in a theoretical setting [27, 28], effect handlers have gained practical interest with several prototype implementations in the form of libraries, interpreters, compilers and runtime representations $[4,5,9,12,15,16,20,21]$.

However, the application space of effect handlers remains largely unexplored. In this paper we explore the applicability of effect handlers to concurrent system

$\triangle$ sk826@cl.cam.ac.uk 
programming in Multicore OCaml. While Multicore OCaml supports sharedmemory parallel programming, this paper restricts its focus to concurrency i.e. overlapped execution of tasks, leaving parallelism outside our scope.

\section{Motivation}

Multicore OCaml [8] incorporates effect handlers as the primary means of expressing concurrency in the language. The modular nature of effect handlers allows the concurrent program to abstract over different scheduling strategies [9]. Moreover, effect handlers allow concurrent programs to be written in directstyle retaining the simplicity of sequential code as opposed to callback-oriented style (as used by e.g. Lwt [34] and Async [24]). In addition to being more readable, direct-style code tends to be easier to debug; unlike callback-oriented code, direct-style code uses the stack for function calls, and hence, backtraces can be obtained for debugging. Indeed, experience from Google suggests that as well as making the code more compact and easier to understand (particularly important when thousands of developers touch the code), direct-style code can perform as well or better than callback-oriented code [3].

Some of the benefits of direct-style code can be achieved by rewriting directstyle functions into callbacks, using syntactic sugar such as Haskell's do-notation for monads or F\#'s async/await [32]. However, this separates functions which use such rewriting from those that do not, leading to awkward mismatches and code duplication: for instance, Haskell provides mapM, filterm and foldm because the ordinary map, filter and foldl functions do not work with monadic arguments. By contrast, effect handlers do not introduce an incompatible type of function.

In Multicore OCaml, the user-level thread schedulers themselves are expressed as OCaml libraries, thus minimising the secret sauce that gets baked into high-performance multicore runtime systems [31]. This modular design allows the scheduling policy to be changed by swapping out the scheduler library for a different one with the same interface. As the scheduler is a library, it can live outside the compiler distribution and be tailored to application requirements.

However, the interaction between user-level threading systems and the operating system services is difficult. For example, the Unix write() system call may block if the underlying buffer is full. This would be fine in a sequential program or a program with each user-level thread mapped to a unique OS thread, but with many user-level threads multiplexed over a single OS thread, a blocking system call blocks the entire program. How then can we safely allow interaction between user-level threads and system services?

Concurrent Haskell [23], which also has user-level threads, solves the problem with the help of specialised runtime system features such as safe FFI calls and bound threads. However, implementing these features in the runtime system warrants that the scheduler itself be part of the runtime system, which is incompatible with our goal of writing thread schedulers in OCaml. Attempts to lift the scheduler from the runtime system to a library in the high-level language while retaining other features in the runtime system lead to further complications [31]. 
Our goals then are:

- Retain the simplicity of direct-style code for concurrent OCaml programs.

- Allow user-level thread schedulers to be written in OCaml as libraries.

- Allow safe interaction between user-level threads and the operating system.

- Perform as well as or better than existing solutions.

We observe that algebraic effects and their handlers can meet all of these goals. In particular, we introduce asynchronous effects and their handlers, and show how they elegantly solve the interaction between user-level threads and operating system services. This paper makes the following contributions:

- We introduce effect handlers for Multicore OCaml and illustrate their utility by constructing a high-performance asynchronous I/O library that exposes a direct style API (Section 3).

- We show how asynchronous effects provide a clean interface to difficult-touse operating system services, such as signal handling and asynchronous notification of I/O completion, and demonstrate how effect handlers enable scoped interrupt handling (Section 4).

- We evaluate the performance of effect handlers in OCaml by implementing a highly scalable web server and show that Multicore OCaml effect handlers are efficient (Section 5).

After the technical content of the paper in Sections 3, 4, and 5, we discuss related work in Section 6 and our conclusions in Section 7.

\section{$3 \quad$ Algebraic effects and their handlers}

Since the primary motivation for adding effect handlers in Multicore OCaml is concurrency, we introduce effect handlers in constructing an asynchronous I/O library which retains the simplicity of direct-style programming ${ }^{4}$.

\subsection{Concurrency}

We start with an abstraction for creating asynchronous tasks and waiting on their results. We use the term fiber to indicate a lightweight user-level thread to distinguish it from kernel threads.

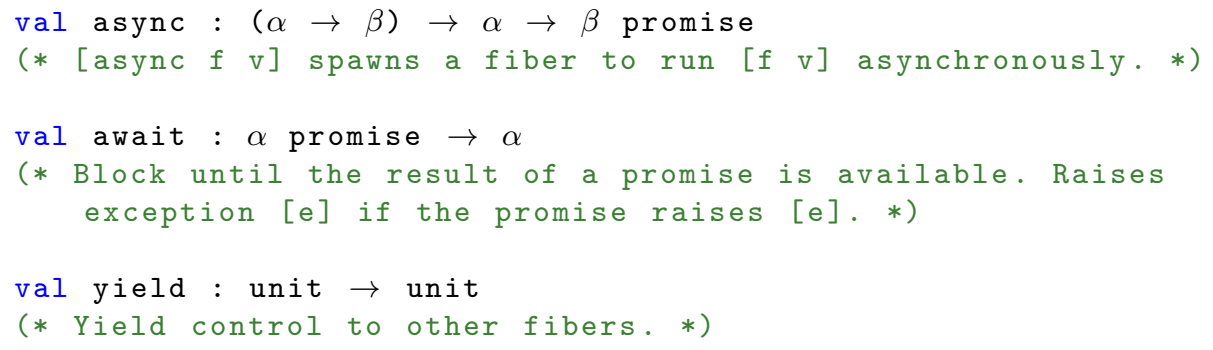

${ }^{4}$ A comprehensive list of example programs written using effect handlers in Multicore OCaml is available at https://github.com/kayceesrk/effects-examples 
Multicore OCaml extends OCaml with the ability to declare user-defined effects with the help of the effect keyword. Since async, await and yield are effectful operations, we declare them as follows:

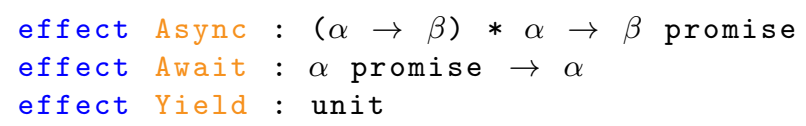

The first declaration says that Async is an effect which is parameterised by a pair of a thunk and a value, and returns a promise as a result when performed. Await is parameterised by a promise and returns the result. Yield is a nullary effect that returns a unit value. To be precise, these declarations are operations of a single built-in effect type $\alpha$ eff in Multicore OCaml. Indeed, these declarations are syntactic sugar for extending the built-in extensible variant type $\alpha$ eff:

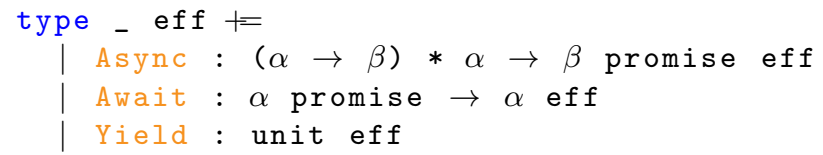

Effects are performed with the perform : $\alpha$ eff $\rightarrow \alpha$ primitive, which performs the effect and returns the result. We can now define the functions async, await and yield as:

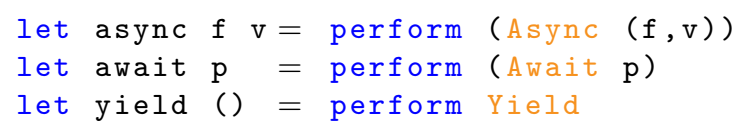

These effects are interpreted by an effect handler, as shown in Figure 1. A promise (lines 1-6) is either completed successfully Done $\mathrm{v}$, failed with an exception Error e or still pending Waiting 1, with a list of fibers waiting on it for completion. The function run (line 8) is the top-level function that runs the main concurrent program. run_q is the queue of concurrent fibers ready to run. The effect handler itself is defined in the lines 17-38. An effect handler comprises of five clauses - a value clause, an exception clause, and three clauses that handle the effects Async, Await and Yield.

Effect clauses are of the form effect $\mathrm{e} k$ where $\mathrm{e}$ is the effect and $\mathrm{k}$ is the continuation of the corresponding perform delimited by this handler. $\mathrm{k}$ is of type $(\alpha, \beta)$ continuation, representing a continuation waiting for a value of type $\alpha$ and returning a value of type $\beta$ when resumed. There are two primitives operating on continuations: continue $\mathrm{k} \times$ resumes the continuation $\mathrm{k}$ where it left off, returning the value $\mathrm{x}$ from perform, while discontinue $\mathrm{k}$ exn resumes the continuation $\mathrm{k}$ by raising the exception exn from perform.

In the case of an Async (f,v) effect (lines 28-31), we create a new promise value $\mathrm{p}$ which is initially waiting to be completed. We set up the original fibers, represented by continuation $\mathrm{k}$, to resume with the promise using the continue primitive. Finally, we recursively call fork to run the new fiber $f$ v. Since Multicore OCaml uses so-called deep handlers, the continuation $\mathrm{k}$ references its surrounding handler, and so we need not write another match expression when continue-ing k (See Kammar et al. [15] for more on deep vs. shallow handlers). 


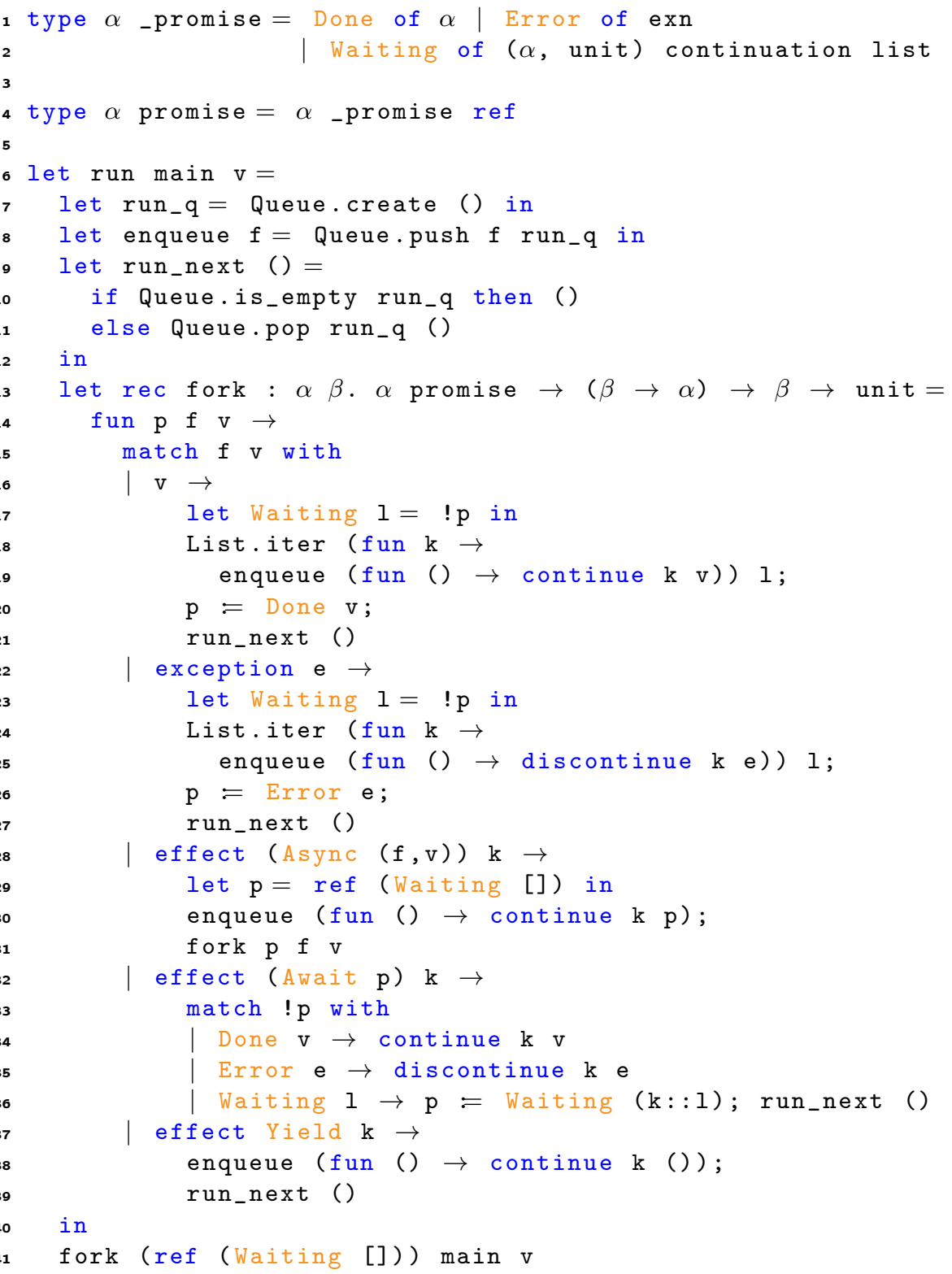

Fig. 1: A simple scheduler, implemented with effects 
In the case of Await p, we check whether the promise is complete. If successful, we immediately resume with the value, and if failed, we use the discontinue primitive to resume the continuation by raising an exception. Otherwise, we block the current fiber on the promise and resume the next fiber from the scheduler. In the case of Yield effect, we enqueue the current fiber and run the next available fiber. In the case of a fiber successfully running to completion (lines 18-23) or raising an exception (lines 24-29), we update the promise, wake up the waiting fibers and resume the next available fiber.

\subsection{Implementing effect handlers}

Unlike other languages that incorporate effect handlers, effects in Multicore OCaml are unchecked. That is, there is no static check for whether all the possible effects have been handled in the program. As a result, a fiber that performs an unhandled effect is discontinued with Unhandled exception.

There are several alternatives to implement the continuations in effect handlers including free monadic interpretations [16, 17, 36], CPS translations [13, 20], and runtime strategies. Multicore OCaml chooses the latter and uses a custom stack layout, efficiently supported by the runtime system. We observe that many effect handlers do not resume the continuations more than once, and support only linear continuations by default, which can be implemented efficiently [9]. We also support explicit copying for non-linear use of continuations.

\subsection{Adding I/O}

Next let us add support for the following I/O operations:

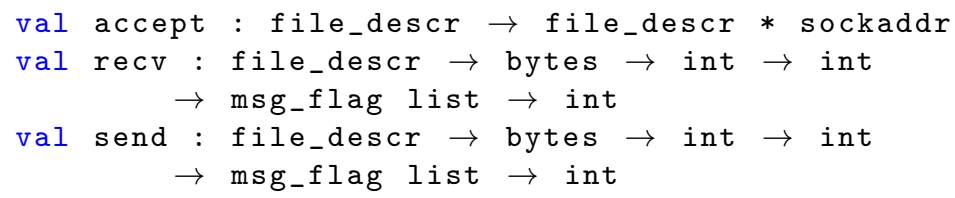

These functions have the same signature as their counterparts in the Unix module. However, invoking any of these functions may block the kernel thread until the I/O operation is complete. In a user-level threaded system this would block the scheduler, preventing other fibers from running.

The standard solution to this problem is to use an event loop, suspending each task performing a blocking I/O operation, and then multiplexing the outstanding I/O operations through an OS-provided blocking mechanism such as select, epoll, kqueue, IOCP, etc. Such asynchronous, non-blocking code typically warrants callback-oriented programming, making the continuations of I/O operations explicit through explicit callbacks (à la JavaScript) or concurrency monad (Lwt and Async libraries for OCaml). The resultant code is arguably messier and more difficult to understand than direct-style code.

Effect handlers lets us retain direct-style while still allowing the use of event loops. Below, we shall just consider accept. The other functions are similar. As 
earlier, we start by declaring an effect for an accept function: effect Accept : file_descr $\rightarrow$ (file_descr * sockaddr). The handler for Accept is:

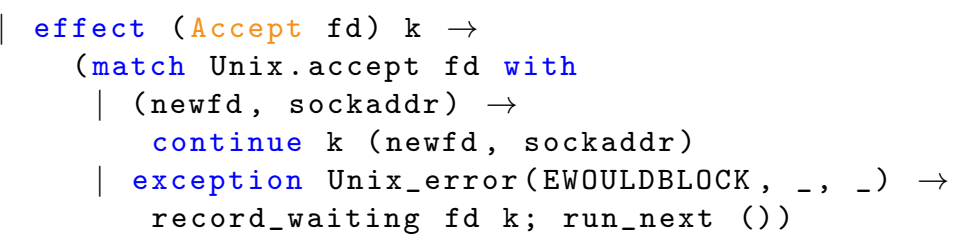

If there is a waiting connection, Unix.accept returns it and we resume the continuation. If not, Unix . accept raises the EWOULDBLOCK error, and we record that the fiber is waiting to accept and switch to the next thread from the scheduler queue. The send and recv operations have similar handler implementations.

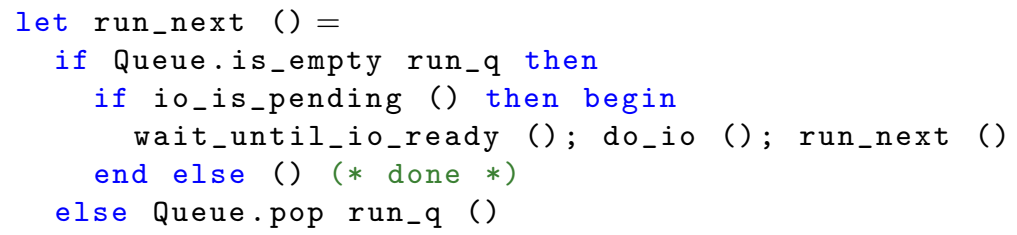

Correspondingly, the run_next function is updated such that it first runs all the available threads, and then if any $\mathrm{I} / \mathrm{O}$ is pending it waits until at least one of the $\mathrm{I} / \mathrm{O}$ operations is ready, and then tries to perform the $\mathrm{I} / \mathrm{O}$ and continue. If the scheduler queue is empty, and there is no pending $\mathrm{I} / \mathrm{O}$, then the scheduler returns. The library blocks on $\mathrm{I} / \mathrm{O}$ only if there are no ready threads and there are pending $\mathrm{I} / \mathrm{O}$ operations.

Using this API, we can write a simple server that echoes client messages:

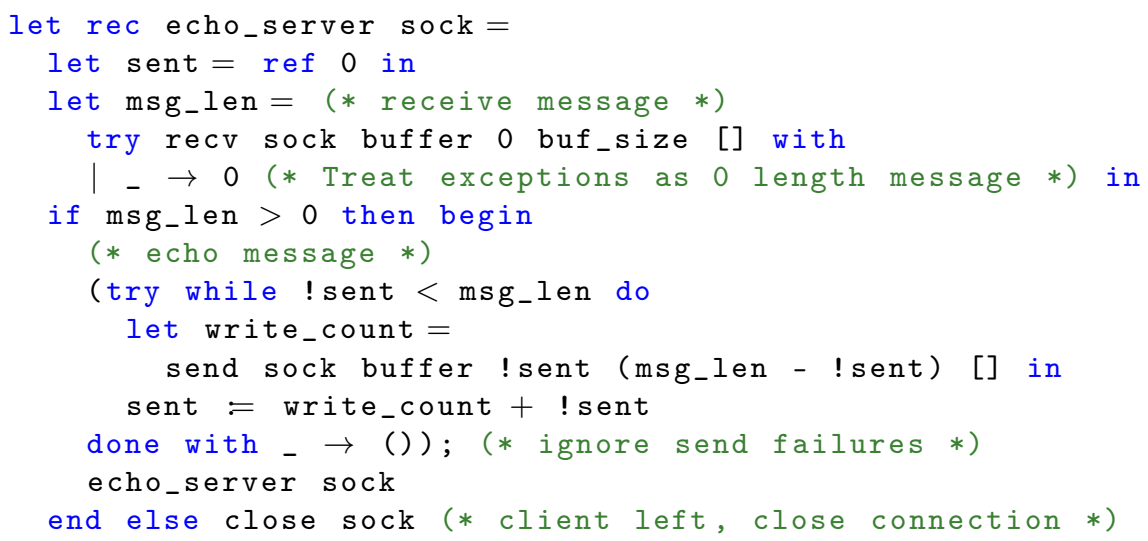

The details of the code are not important, but observe that the code is in direct-style and moreover is the same code for the synchronous, blocking echo server. Furthermore, since the following code is asynchronous, the two echo servers on sock1 and sock2 do not block each other:

$\operatorname{run}(\operatorname{fun}() \rightarrow$

async echo_server sock1; async echo_server sock2) () 


\subsection{Default handlers}

For an invocation of an effectful operation to be meaningful it must happen in the scope of an appropriate handler. A default handler is a convenient mechanism for ensuring that an operation invocation is always meaningful even when not in scope of a handler. A default handler provides a default interpretation of an operation. This interpretation is chosen if no other appropriate handler encloses the invocation context. In other words, a default handler can operationally be understood as a top level handler which encloses the entire program context including itself. As a concrete example we can give a default synchronous semantics for Accept

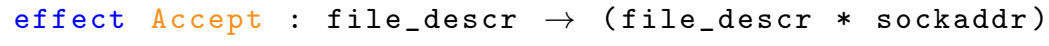

In Multicore OCaml a default handler is declared along with the effectful operation it is handling using the familiar function construct. In contrast to a regular effect handler, a default handler does not expose the continuation of the operation to the programmer, rather, the continuation is implicitly applied to the body clause(s). This particular design admits an efficient implementation, since every continuation invocation in a default handler is guaranteed to be in tail position. Thus the runtime does not need to allocate a continuation, it can simply return the value produced by the default handler clause. As a consequence an invocation of a default handler amounts to a function call. This makes it possible for effectful libraries to remain performance backwards compatible with programs that do not use regular effect handlers.

Continuing, we can also obtain the synchronous counterparts to Await, Async, and Yield by giving them all a default synchronous semantics, i.e.

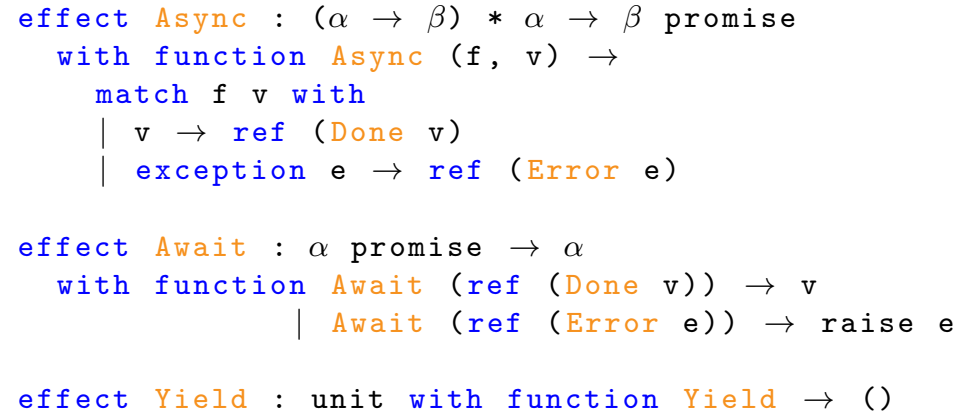

If a default handler raises an exception, then the fiber is discontinued with that exception. Furthermore, if a default handler performs an effect then the default handler of that effect is invoked. If we define the default implementations of Send and Recv in a similar way then by using default handlers the following program behaves exactly like its synchronous counterpart.

async echo_server sock1; async echo_server sock2 


\section{Programming with resources and effects}

Systems programming generally involves the manipulation of scarce resources such as file handles, connections and locks. Such resources are inherently linear, stateful values: once a file handle is closed, it cannot be used again.

Ordinary straight-line imperative code is not enough to use resources correctly in the presence of exceptions, let alone effects. For instance, the following code leaks an unclosed file handle if do_stuff_with $f$ raises an exception:

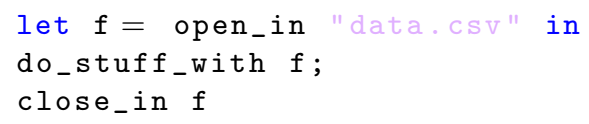

We need to ensure that the file handle is closed, even if an exception is raised:

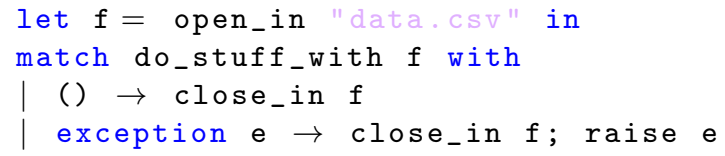

Note that the initial open_in occurs outside the exception handler - if opening the file fails with an exception, we need not close it. This idiom or something equivalent is widespread, often with syntactic support as try-finally.

However, note an implicit assumption in this code, that if do_stuff_with $f$ terminates then it does so only once. If the computation do_stuff_with $f$ were to return twice (by allowing a continuation captured inside $f$ to be resumed twice), then the cleanup code (close_in $f$ in this example) would incorrectly run multiple times. If the computation do_stuff_with_f were to continue execution after the cleanup code had run, its operations would have unexpected effects.

As well as the performance advantages mentioned above, this is the other major reason that our continuations are linear. By preserving the linearity of computations (operations that are begun once do not complete twice), we allow resource-manipulating code to work correctly in the presence of effects.

Some interesting examples of programming with effects and handlers (such as backtracking) are incompatible with this approach, since they rely on continuations to be usable more than once. To support experimenting with such examples, we do provide a primitive to allow re-use of continuations, with the proviso that it is not safe in general when used with code that handles resources.

The linearity of computations is implicit in OCaml without effect handlers, but once continuations appear as first-class values the possibility of using them twice arises. OCaml does not have the linear types necessary to prevent this statically (and we are not proposing to add them), so we must enforce linearity dynamically. Ensuring that a continuation is not used twice is easy enough, by keeping a bit of state in the continuation, updated by continue and discontinue so that subsequent resumptions fail. Ensuring that a continuation is not simply discarded is harder: the system must detect when a continuation is being garbage-collected, and discontinue it with a special exception so that resource cleanup code runs. 


\subsection{Asynchronous exceptions}

Correct use of resources is much more difficult in the presence of asynchronous exceptions. For example, on Unix-like systems when the user of a command-line program presses Ctrl-C the SIGINT signal is sent. By default, this terminates the program. However, programs may indicate that they can handle this signal, for instance by cancelling a long-running task and accepting user input again.

In OCaml, programs indicate willingness to handle SIGINT by calling Sys .catch_break true. From that point onwards, the special exception Sys.Break may be raised at essentially any point in the program, if the user presses Ctrl-C. Unfortunately, the try-finally idiom does not clean up correctly in this case:

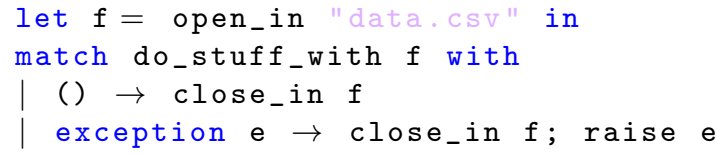

If Sys.Break is raised just after open_in returns but before the match statement is entered, then the file handle will never be closed. To eliminate this possibility, we need to temporarily disable asynchronous exceptions. Suppose we introduce two functions set_mask and clear_mask, to disable (mask) and re-enable asynchronous exceptions. Our second attempt at resource handling looks like:

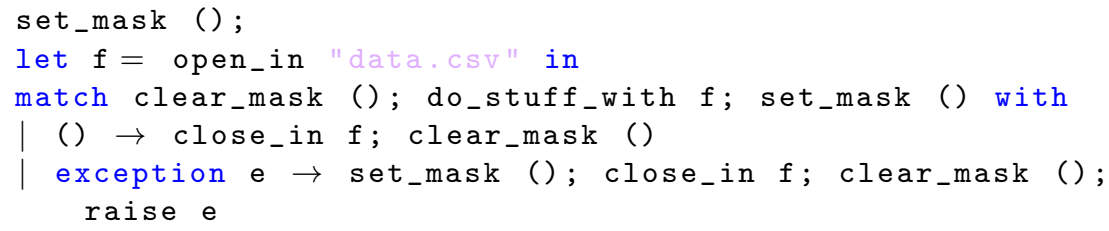

Correctly placing calls to set_mask and clear_mask is a very tricky business. Indeed, the above code has a serious bug: if open_in fails with an ordinary synchronous exception (because e.g. the file is not found), then asynchronous exceptions will never be unmasked.

Instead, we follow the good advice of Marlow et al. in the design of Haskell's asynchronous exceptions [22], and prefer instead scoped combinators:

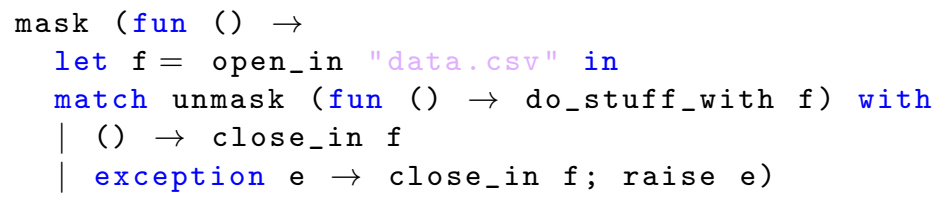

The changes to the masking state made by mask and unmask apply only to one scope, and are automatically undone, making it impossible to accidentally leave asynchronous exceptions masked. The presence of unmask allows a further improvement to the semantics: we model the asynchronous exception Sys. Break as being raised not by whatever code happened to be executing when Ctrl-C was pressed, but by the nearest enclosing unmask. This ensures that exception handlers for asynchronous exceptions need not be treated specially. In particular, the follow code cannot fail, no matter when Ctrl-C is pressed: 


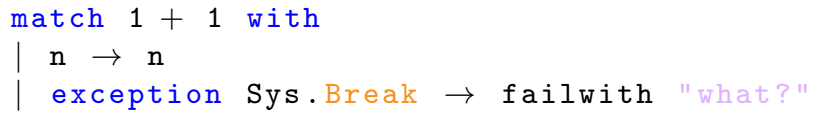

\subsection{Signal handling and asynchronous effects}

Even with the scoped masking combinators, it is difficult and unpleasant to write code that correctly manipulates resources in the presence of asynchronous exceptions. For this reason, many systems choose instead to poll for cancellation requests, instead of being interrupted with one. That is, instead of a Sys.Break exception being raised, the programmer manually and regularly checks a mutable boolean cancellation_flag, which is asynchronously set to true when the user presses Ctrl-C (This check may be combined with other system facilities: one common choice is that cancellation is checked at all $\mathrm{I} / \mathrm{O}$ operations, since the program must handle failures there anyway).

On Unix-like systems, it is possible to implement this behaviour using a signal handler, which is a callback invoked when a signal is raised (e.g. by the user pressing Ctrl-C). In OCaml, these can be installed using the function Sys. set_signal. In fact, the behaviour of the previously-mentioned Sys.catch_break is implemented by installing a signal handler that raises Sys. Break:

set_signal sigint (Signal_handle(fun _ $\rightarrow$ raise Break))

Synchronous cancellation can be implemented using a signal handler that sets a cancellation flag:

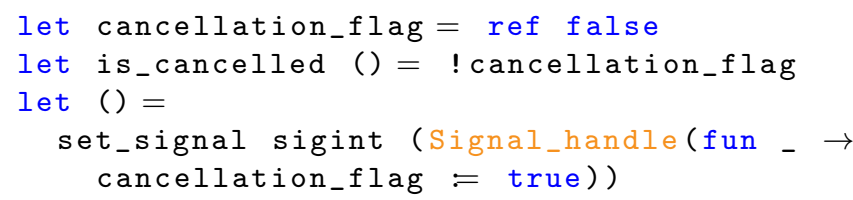

By removing the possibly of cancellation except at designated points, the imperative parts of the system become safer and easier to write. However, as Marlow et al. [22] note, for the purely functional parts of the system asynchronous cancellation is both necessary and just as safe as synchronous: necessary, because inspecting the mutable cancellation state breaks referential transparency, and safe, because purely functional code holds no resources and pure computations can be abandoned without issue.

In order to call a pure function from imperative code while maintaining prompt cancellation, we need to switch from synchronous (polling) cancellation to asynchronous cancellation and back, by providing a combinator:

async_cancellable $:($ unit $\rightarrow \alpha) \rightarrow$ (unit $\rightarrow \alpha$ option)

Normally, async_cancellable $f$ returns Some $(f())$. However, the computation $f$ may be cancelled asynchronously, causing async_cancellable $f$ to return None, ensuring that asynchronous cancellation does not affect the caller.

Our first attempt at such a mechanism looks like: 


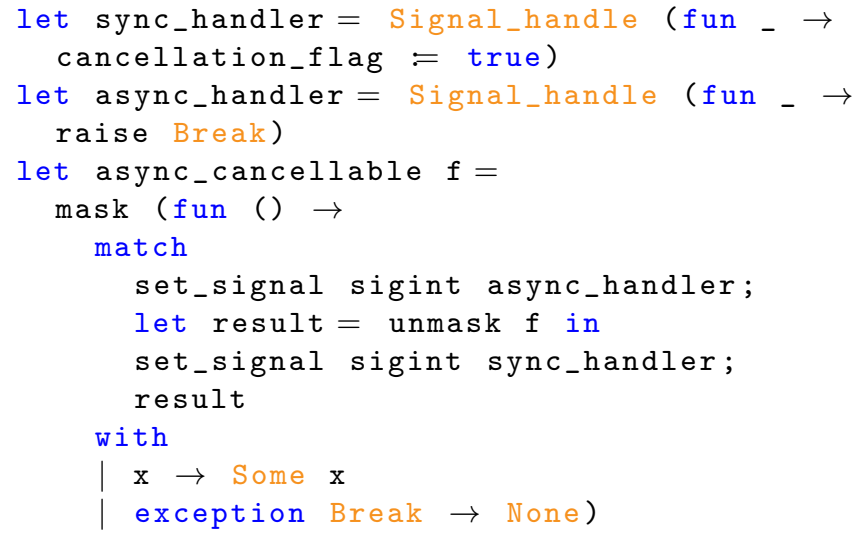

This code is tricky, due to its delicate mutation of global state. It is very similar to the code we saw earlier using set_mask and clear_mask, and even has the same bug: it leaves the wrong signal handler in place if $\mathrm{f}$ raises an exception.

As before, scoped combinators make such code easier to get right (or, more accurately, harder to get wrong). To this end, we introduce asynchronous effects, which are effects that can be performed asynchronously, just as asynchronous exceptions can be raised asynchronously. By treating Break as an asynchronous effect, we can mix synchronous and asynchronous cancellation reliably.

For synchronous cancellation, we handle the Break effect by setting a flag:

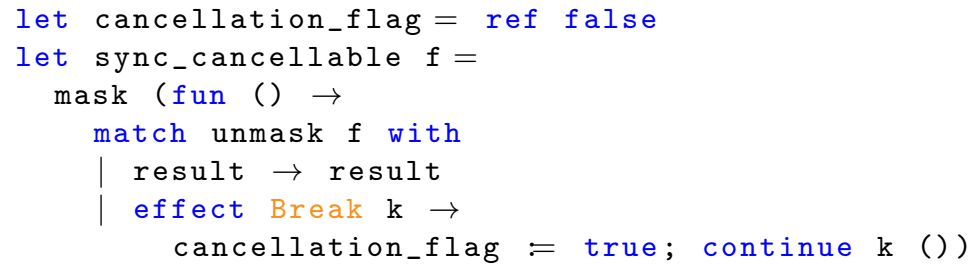

Asynchronously-cancellable code can be implemented by handling the Break effect and discarding the continuation. Since effect handlers delimit the continuation, the asynchrony is limited to the specified function.

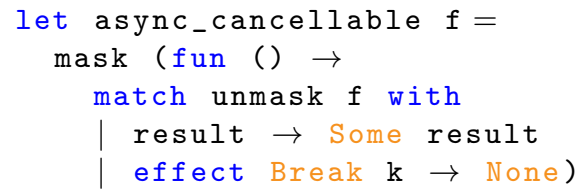

Instead of having a single global callback as the current signal handler, asynchronous effects allow handlers to delimit their scope and nest correctly.

\subsection{Managing multiple computations with asynchronous effects}

Unlike signal handlers, asynchronous effects get an explicit representation of the computation they interrupted by way of the continuation $\mathrm{k}$. While signal handlers 
can only resume the computation or abandon it (by raising an exception), effect handlers have other options available. For instance, a scheduler which maintains a collection of tasks can switch to another task when handling an asynchronous effect, just as the scheduler in Fig. 1 does for the synchronous Yield effect. Using asynchronous effects the cooperative scheduler of Fig. 1 can be made preemptive, by asking the operating system to provide a periodic timer signal (using e.g. the Unix timer_create API), and adding a new clause to the scheduler:

| effect TimerTick $k \rightarrow$ enqueue (continue $k$ ); run_next()

\subsection{Asynchronous I/O notifications}

Operating systems provide several different I/O interfaces. The simplest is the direct-style blocking $I / O$, in which the program calls operating-system functions which do not return until the operation completes. This allows a straightforward style of programming in which the sequence of I/O operations matches the flow of the code. We aim to support this style of programming using alternative operating system interfaces that allow multiple I/O operations to be overlapped.

In Section 3.3, we saw one way of accomplishing this with effects, by using multiplexing mechanisms like select, poll, etc., which block until one of several file descriptors is ready. An alternative is asynchronous $I / O$, in which multiple operations are submitted to the operating system, which overlaps their execution. However, applications written using asynchronous $\mathrm{I} / \mathrm{O}$ tend to have complex control flow which does not clearly explain the logic being implemented, due to the complexity of handling the operating system's asynchronous notifications of $\mathrm{I} / \mathrm{O}$ completion.

We propose effects and handlers as a means of writing direct-style I/O code, but using the asynchronous operating system interfaces. We introduce two new effect operations: Delayed, which describes an operation that has begun and will complete later, and Completed, which describes its eventual completion. Both of these take an integer parameter, which identifies the particular operation.

Potentially long-running operations like read perform the Delayed effect, indicating that the operation has been submitted to the operating system but has not yet completed. Later, upon receipt of an operating-system completion notification, the asynchronous effect Completed is performed.

Using this mechanism, support for asynchronous completions can be added to the scheduler of Fig. 1 by adding clauses for the Delayed and Completed effects, where ongoing_io is an initially empty hash table:

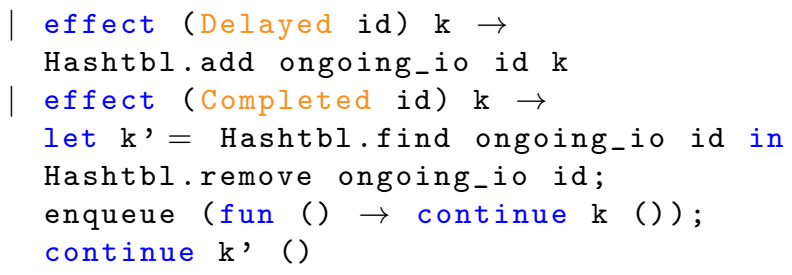


In this sample, the continuation $\mathrm{k}$ of the Delayed effect is the continuation of the code performing the I/O operation, which instead of being immediately invoked is stored in a hash table until it can be invoked without blocking.

The continuation $\mathrm{k}$ of the Completed effect is the continuation of whichever fiber was running when the $\mathrm{I} / \mathrm{O}$ completed. This scheduler chooses to preempt that fiber in favour of the fiber that performed the I/O. Equally, the scheduler could give priority to the running fiber, by swapping $\mathrm{k}$ and $\mathrm{k}$ ' in the last lines.

\section{Results}

So far we have presented what we believe are compelling applications of effect handlers for elegant system programming. However, none of that would matter if the resultant programs were unacceptably slower compared to extant solutions. Hence, in this section, we evaluate the performance of a web server built with effect handlers against existing production-quality web servers.

We have implemented an effect-based asynchronous I/O library, aeio [2], that exposes a direct-style API to the clients. At its core, aeio uses the main loop engine from the Lwt library using the libev $^{5}$ event loop (using epoll in our experiments). For the OCaml web server, we use httpaf, which is a high performance, memory efficient, and scalable web server that uses the Async library [24] (also using epoll as its I/O system call). We then extended httpaf and implemented an effect handler based backend using aeio. The configurations we use for the evaluation are:

- Effect: Effect-based version which uses httpaf with aeio on the Multicore OCaml compiler. The Multicore OCaml compiler was forked off vanilla OCaml version 4.02.2.

- Async: Vanilla OCaml version 4.03.0, using httpaf + Async 113.33.03.

- Go: Go 1.6.3 version of the benchmark using net/http package.

For comparison, all three configurations were constrained to only one core (using the GOMAXPROCS variable in the case of Go).

The evaluations were done on a $3 \mathrm{GHz}$ Intel Core i7 with $16 \mathrm{~GB}$ of main memory running 64-bit Ubuntu 16.10. The client workload was generated by the wrk ${ }^{6}$ program. Each wrk2 run uses a fixed number of client connections that issues requests at a constant rate, and measures request latency and throughput.

Figure 2 shows the latency profiles for 1 minute runs under two different configurations. At $1 \mathrm{k}$ connections and $10 \mathrm{k}$ requests per second, the effect implementation performs marginally better than Async. Go performs the best with all requests satisfied within $27 \mathrm{~ms}$. The average request latency of effect configuration is $2.127 \mathrm{~ms}$ over 587969 requests. Under this configuration, the observed throughput is between 9780 and 9800 requests per second in all of the configurations.

\footnotetext{
${ }^{5}$ http://software.schmorp.de/pkg/libev.html

${ }^{6}$ https://github.com/giltene/wrk2
} 


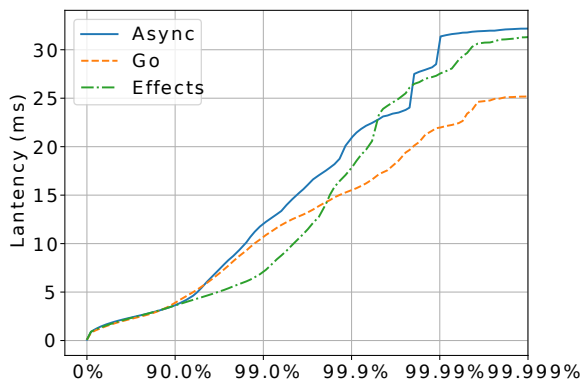

(a) Medium contention: $1 \mathrm{k}$ connections, $10 \mathrm{k}$ requests/sec

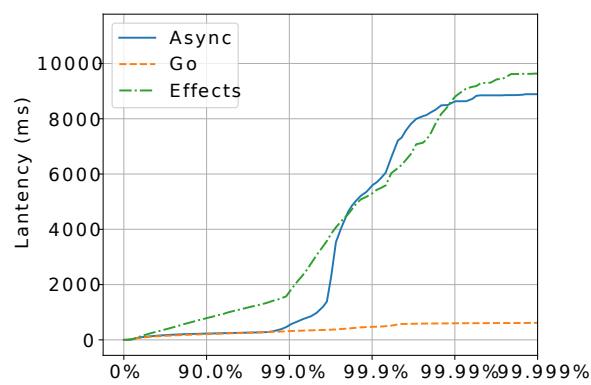

(b) High contention: $10 \mathrm{k}$ connections, $30 \mathrm{k}$ requests $/ \mathrm{sec}$

Fig. 2: Latency profile of client requests

At high loads, the performance degrades substantially in every configuration, but it is worse in the OCaml implementations. The average latency for satisfying a client request increases to $333.40 \mathrm{~ms}$ in the effect case, while it is $139 \mathrm{~ms}$ in async and $107.25 \mathrm{~ms}$ in Go. While Go achieved 17389 requests per second, Async and effect implementations achieved only 16761 and 15440 requests per second, respectively. This indicates that there is room for optimisations. Multicore OCaml has a new garbage collector, which has not been tuned to the extent of vanilla OCaml and Go. We strongly suspect that garbage collector optimisation and tuning would lead to performance improvements. Importantly, the tail latencies of both OCaml implementations (the vanilla Async and our effectbased server) were comparable in both configurations, indicating that there is no significant performance degradation from our switch to using the effects model presented in this paper.

\section{Related work}

Implementations of effect handlers Since their inception, several implementations of algebraic effect handlers have appeared, many of which are implemented as libraries in existing programming languages $[5,15,16,17,18,30,36]$. There are several other implementations that like Multicore OCaml provide language level support for effect handlers:

- Eff [4] is the first programming language designed with effect handlers in mind. It is a strict language with Hindley-Milner type inference similar in spirit to ML. It includes a novel feature for supporting fresh generation of effects in order to support effects such as ML-style higher-order state. Eff compiles to a free monad embedding in OCaml [29].

- Frank [21] is a programming language with effect handlers but no separate notion of function: a function is but a special case of a handler. Frank has a bidirectional type and effect system with a novel form of effect polymorphism. Furthermore, the handlers in Frank are so-called shallow handlers, 
which do not implicitly wrap themselves around the continuation, thereby allowing nonuniform interpretations of operations.

- Koka is a functional web-oriented programming language which has recently been enriched with effect handlers [20]. It has a type-and-effect system which is based on row polymorphism. Koka uses a novel type-and-effect driven selective CPS compilation scheme for implementing handlers on managed platforms such as .NET and JavaScript.

- Links [7] is a single source, statically typed language with effect tracking for multi-tier web programming. Links supports effect handlers on both the client and the server. The server side implementation is based on a generalised abstract CEK machine [12], while the client side implementation is based on a CPS translation [13]. Links also has a prototype compiler for the server side with effect handlers based on the Multicore OCaml compiler [14].

A common theme for the above implementations is that their handlers are multi-shot handlers which permit multiple invocations of continuations.

Asynchronous IO Many systems seek to combine the simplicity of direct-style, blocking I/O with the performance gains of allowing independent operations to complete in parallel. Indeed, the blocking I/O interfaces of most operating systems are designed in this way, by descheduling a process that requests a slow operation and running another process until the operation completes. However, operating system mechanisms rely on hardware context switching. The high overheads of such mechanisms lead to a desire for lightweight concurrent tasks integrated into programming languages.

The Erlang system [33] is a good example, capable of managing large numbers of lightweight processes with an integrated scheduler, and multiplexing their I/O onto operating system interfaces like select, poll, etc. More recently, the work by Syme et al. [32] adding async/await to $\mathrm{F} \#$ allows the programmer to specify which operations should be completed asynchronously, implemented by compiling functions which use async differently from those that do not. The work by Marlow et al. on Concurrent Haskell [23] also supports large numbers of concurrent threads with multiplexed I/O, while allowing possibly-blocking operating system services to be used without blocking the entire system via the mechanism of safe foreign calls. Leijen [19] describes an implementation of a fullfledged async-await library implemented using effect handlers in Koka including cancellation and timeout. Koka compiles to JavaScript, whose concurrency model is cooperative. In particular, there are no asynchronous interrupts in JavaScript and Koka does not need the associated machinery to safely handle them.

Resource handling with control operators Programming languages supporting systems programming and exceptions generally support some variant of the tryfinally idiom, often with syntactic support. For example, $\operatorname{try}\{\ldots\}$ finally $\{\ldots\}$ in Java, using statements in C\#, destructors and RAII in $\mathrm{C}++$, or defer in Go.

Languages with more powerful control operators require correspondingly more powerful constructs for safe resource handling. The Common LISP condition system allows conditions (similar to effects) to be handled by abandoning 
the computation with an error, restarting it or ignoring the error and continuing, but does not allow the continuation to be captured as a value. It supports the unwind-protect form to ensure that cleanup code is run no matter how a block is exited. See Pitman [26] for an analysis of the condition system's design.

Scheme supports general nonlinear continuations [1], which present difficulties when handling inherently linear resources. Many Scheme implementations provide a primitive dynamic-wind [11], which generalises the try-finally idiom by taking some setup and cleanup code to be run not just once but every time control passes in and out of a specified block of code. However, this comes with its own caveats: the naive approach of using dynamic-wind to open and close a file will close and reopen the file every time a computation is paused and resumed, which is not safe in general as the file may not still exist at the second opening. One-shot (linear) continuations have also been proposed for Scheme [6].

Support for truly asynchronous interrupts is more rare, partially due to the difficulty of programming in their presence. The Unix signalling mechanism is an important example, but its reliance on global mutable state makes programming difficult (see Section. 4.2). Marlow et al. [22] present a more composable design for asynchronous exceptions in Haskell. Our approach can be viewed as the extension of the Haskell approach to effects as well as exceptions.

\section{Conclusion}

Multicore OCaml provides effect handlers as a means to abstract concurrency. In this paper, we have described and demonstrated the utility of effect handlers in concurrent system oriented programming. We have developed a direct-style asynchronous I/O with effect handlers [2]. Using this library, we built a highly concurrent and scalable web server. Our evaluation shows that this implementation retains a comparative performance with the current state of the art in vanilla OCaml, but that OCaml has some room for improvement vs direct-style multicore concurrency in Go.

Rather than providing the full generality of effect handlers with nonlinear continuations, our design provides effect handlers with linear continuations. This design admits a particularly efficient implementation. Furthermore, linear continuations interplay more smoothly with system resources. Our implementation of asynchronous effects also provides an elegant solution to handling problematic corner cases in typical operating system interfaces, such as reliable signal handling and efficiently implementing quirky system call interfaces while exposing a simple, portable interface to the developer.

\section{Acknowledgements}

Daniel Hillerström was supported by EPSRC grant CDT in Pervasive Parallelism (EP/L01503X/1). KC Sivaramakrishnan was supported by a Research Fellowship from the Royal Commission for the Exhibition of 1851. 


\section{Bibliography}

[1] Abelson, H., Dybvig, R.K., Haynes, C.T., Rozas, G.J., Adams, N., Friedman, D.P., Kohlbecker, E., Steele, G., Bartley, D.H., Halstead, R., et al.: Revised(5) report on the algorithmic language Scheme. Higher-order and symbolic computation 11(1), 7-105 (1998)

[2] Aeio: An asynchronous, effect-based I/O library (2017), https://github. com/kayceesrk/ocaml-aeio, accessed: 2017-05-05 09:21:00

[3] Barroso, L., Marty, M., Patterson, D., Ranganathan, P.: Attack of the Killer Microseconds. Commun. ACM 60(4), 48-54 (Mar 2017), http://doi.acm. org $/ 10.1145 / 3015146$

[4] Bauer, A., Pretnar, M.: Programming with Algebraic Effects and Handlers. Journal of Logical and Algebraic Methods in Programming 84(1), 108-123 (2015)

[5] Brady, E.: Programming and Reasoning with Algebraic Effects and Dependent Types. In: Proceedings of the 18th ACM SIGPLAN International Conference on Functional Programming. pp. 133-144. ICFP '13, ACM, New York, NY, USA (2013), http://doi.acm.org/10.1145/2500365.2500581

[6] Bruggeman, C., Waddell, O., Dybvig, R.K.: Representing Control in the Presence of One-Shot Continuations. In: Fischer, C.N. (ed.) Proceedings of the ACM SIGPLAN'96 Conference on Programming Language Design and Implementation (PLDI), Philadephia, Pennsylvania, May 21-24, 1996. pp. 99-107. ACM (1996)

[7] Cooper, E., Lindley, S., Wadler, P., Yallop, J.: Links: Web Programming Without Tiers. In: Proceedings of the 5th International Conference on Formal Methods for Components and Objects. pp. 266-296. FMCO'06, Springer-Verlag, Berlin, Heidelberg (2007), http://dl.acm. org/citation.cfm?id=1777707.1777724

[8] Dolan, S., White, L., Madhavapeddy, A.: Multicore OCaml. OCaml Workshop (2014)

[9] Dolan, S., White, L., Sivaramakrishnan, K., Yallop, J., Madhavapeddy, A.: Effective Concurrency through Algebraic Effects. OCaml Workshop (2015)

[10] Forster, Y., Kammar, O., Lindley, S., Pretnar, M.: On the Expressive Power of User-defined Effects: Effect Handlers, Monadic Reflection, Delimited Control. Proc. ACM Program. Lang. 1(ICFP), 13:1-13:29 (Aug 2017), http://doi.acm.org/10.1145/3110257

[11] Friedman, D.P., Haynes, C.T.: Constraining Control. In: Proceedings of the 12th ACM SIGACT-SIGPLAN Symposium on Principles of Programming Languages. pp. 245-254. POPL '85, ACM, New York, NY, USA (1985), http://doi.acm.org/10.1145/318593.318654

[12] Hillerström, D., Lindley, S.: Liberating Effects with Rows and Handlers. In: Proceedings of the 1st International Workshop on Type-Driven Development. pp. 15-27. TyDe 2016, ACM, New York, NY, USA (2016), http://doi.acm.org/10.1145/2976022.2976033 
[13] Hillerström, D., Lindley, S., Atkey, R., Sivaramakrishnan, K.C.: Continuation Passing Style for Effect Handlers. In: Miller, D. (ed.) 2nd International Conference on Formal Structures for Computation and Deduction (FSCD 2017). Leibniz International Proceedings in Informatics (LIPIcs), vol. 84, pp. 18:1-18:19. Schloss Dagstuhl-Leibniz-Zentrum fuer Informatik, Dagstuhl, Germany (2017), http://drops.dagstuhl.de/opus/ volltexte/2017/7739

[14] Hillerström, D., Lindley, S., Sivaramakrishnan, K.: Compiling Links Effect Handlers to the OCaml Backend. ML Workshop (2016)

[15] Kammar, O., Lindley, S., Oury, N.: Handlers in action. In: Proceedings of the 18th ACM SIGPLAN International Conference on Functional Programming. pp. 145-158. ICFP '13, ACM, New York, NY, USA (2013), http://doi.acm.org/10.1145/2500365.2500590

[16] Kiselyov, O., Ishii, H.: Freer Monads, More Extensible Effects. In: Proceedings of the 2015 ACM SIGPLAN Symposium on Haskell. pp. 94-105. Haskell '15, ACM, New York, NY, USA (2015), http://doi . acm.org/10. $1145 / 2804302.2804319$

[17] Kiselyov, O., Sabry, A., Swords, C.: Extensible Effects: An Alternative to Monad Transformers. In: Proceedings of the 2013 ACM SIGPLAN Symposium on Haskell. pp. 59-70. Haskell '13, ACM, New York, NY, USA (2013), http://doi.acm.org/10.1145/2503778.2503791

[18] Kiselyov, O., Sivaramakrishnan, K.: Eff directly in OCaml. ML Workshop (2016)

[19] Leijen, D.: Structured Asynchrony with Algebraic Effects. In: Proceedings of the 2Nd ACM SIGPLAN International Workshop on Type-Driven Development. pp. 16-29. TyDe 2017, ACM, New York, NY, USA (2017), http://doi.acm.org/10.1145/3122975.3122977

[20] Leijen, D.: Type Directed Compilation of Row-typed Algebraic Effects. In: Proceedings of the 44th ACM SIGPLAN Symposium on Principles of Programming Languages. pp. 486-499. POPL 2017, ACM, New York, NY, USA (2017), http://doi.acm.org/10.1145/3009837.3009872

[21] Lindley, S., McBride, C., McLaughlin, C.: Do be do be do. In: Proceedings of the 44th ACM SIGPLAN Symposium on Principles of Programming Languages. pp. 500-514. POPL 2017, ACM, New York, NY, USA (2017), http://doi.acm.org/10.1145/3009837.3009897

[22] Marlow, S., Jones, S.P., Moran, A., Reppy, J.: Asynchronous Exceptions in Haskell. In: Proceedings of the ACM SIGPLAN 2001 Conference on Programming Language Design and Implementation. pp. 274-285. PLDI '01, ACM, New York, NY, USA (2001), http://doi.acm.org/10.1145/ 378795.378858

[23] Marlow, S., Jones, S.P., Thaller, W.: Extending the Haskell Foreign Function Interface with Concurrency. In: Proceedings of the 2004 ACM SIGPLAN Workshop on Haskell. pp. 22-32. Haskell '04, ACM, New York, NY, USA (2004), http://doi.acm.org/10.1145/1017472.1017479 
[24] Minsky, Y., Madhavapeddy, A., Hickey, J.: Real World OCaml - Functional Programming for the Masses. O'Reilly (2013), http://shop. oreilly.com/ product/0636920024743.do\#tab_04_2

[25] Moggi, E.: Notions of Computation and Monads. Information and Computation 93(1), 55-92 (Jul 1991), http://dx.doi.org/10.1016/ 0890-5401 (91) 90052-4

[26] Pitman, K.M.: Condition Handling in the Lisp Language Family, pp. 39-59. Springer Berlin Heidelberg, Berlin, Heidelberg (2001)

[27] Plotkin, G., Power, J.: Adequacy for Algebraic Effects. In: International Conference on Foundations of Software Science and Computation Structures. pp. 1-24. Springer (2001)

[28] Plotkin, G.D., Pretnar, M.: Handling Algebraic Effects. Logical Methods in Computer Science 9(4) (2013), https://doi.org/10.2168/LMCS-9(4: 23) 2013

[29] Pretnar, M., Saleh, A.H., Faes, A., Schrijvers, T.: Efficient Compilation of Algebraic Effects and Handlers. Tech. Rep. CW 708, KU Leuven, Belgium (Oct 2017)

[30] Saleh, A.H., Schrijvers, T.: Efficient Algebraic Effect Handlers for Prolog. Theory and Practice of Logic Programming 16(5-6), 884-898 (2016)

[31] Sivaramakrishnan, K.C., Harris, T., Marlow, S., Peyton Jones, S.: Composable Scheduler Activations for Haskell. Journal of Functional Programming 26, e9 (2016)

[32] Syme, D., Petricek, T., Lomov, D.: The F\# Asynchronous Programming Model. In: Proceedings of the 13th International Conference on Practical Aspects of Declarative Languages. pp. 175-189. PADL'11, SpringerVerlag, Berlin, Heidelberg (2011), http://dl.acm.org/citation. cfm?id= 1946313.1946334

[33] Virding, R., Wikström, C., Williams, M.: Concurrent Programming in ERLANG (2Nd Ed.). Prentice Hall International (UK) Ltd., Hertfordshire, UK, UK (1996)

[34] Vouillon, J.: Lwt: A Cooperative Thread Library. In: Proceedings of the 2008 ACM SIGPLAN Workshop on ML. pp. 3-12. ML '08, ACM, New York, NY, USA (2008), http://doi.acm.org/10.1145/1411304.1411307

[35] Wadler, P.: The Essence of Functional Programming. In: Proceedings of the 19th ACM SIGPLAN-SIGACT Symposium on Principles of Programming Languages. pp. 1-14. POPL '92, ACM, New York, NY, USA (1992), http: //doi.acm.org/10.1145/143165.143169

[36] Wu, N., Schrijvers, T., Hinze, R.: Effect Handlers in Scope. In: Proceedings of the 2014 ACM SIGPLAN Symposium on Haskell. pp. 1-12. Haskell '14, ACM, New York, NY, USA (2014), http://doi.acm.org/10.1145/ 2633357.2633358 